\title{
Tradução e adaptação cultural do APQ-Aging Perceptions \\ Questionnaire para a língua portuguesa brasileira
}

\section{Transtaionandcrossaltura adaptation of theAPQAging Perceptions Questionaire toBravilianPartuguese}

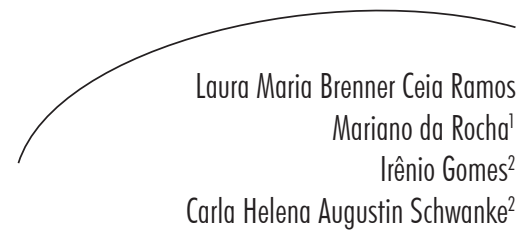

Resumo

O envelhecimento é um fenômeno complexo que envolve as dimensões física, psicológica e sociocultural, incluindo a autopercepção da saúde e do envelhecimento, devendo estas ser abordadas e, portanto, fazer parte da avaliação geriátrica ampla. No caso da autopercepção de saúde, existe vasta literatura nacional e internacional. Contudo, em relação à autopercepção do envelhecimento, os estudos ainda são incipientes, especialmente no Brasil, assim este estudo tem como objetivo apresentar os resultados do processo de tradução e adaptação cultural do Aging Perceptions Questionnaire (APQ) para a língua portuguesa brasileira. Para tanto, foi utilizada a metodologia proposta por Beaton et al., que propôs a realização de duas traduções para o português (T1 e T2), a síntese destas traduções (T12), duas retrotraduções, a síntese destas retrotraduções, a análise pelo comitê de especialistas e a aplicação do pré-teste. A validade de conteúdo foi feita pela análise do comitê e por análise qualitativa do pré-teste. Após a aplicação do pré-teste e entrevista individual com 30 indivíduos, foram realizadas duas alterações no documento T12 usado como pré-teste. O documento final gerado a partir da tradução e adaptação cultural do instrumento original será aplicado posteriormente em uma amostra maior, para sua validação para a população brasileira.

\section{Abstract}

Aging is a complex phenomenon involving physical, psychological and sociocultural dimensions, including the self perception of health and aging. As such, they should be addressed and be part of the general geriatric evaluation. In the case of self perception of health, there is a vast national and international literature. However, concerning self perception of aging, the studies are still very incipient, especially in Brazil, so this paper presents the results of the translation and cross-cultural adaptation process of

Palavras-chave:

Envelhecimento.

Autoimagem. Questionários. Tradução. Autopercepção do envelhecimento.

Instrumentos.
Key words: Aging. Self Concept. Questionnaires. Translating. Instruments.

Programa de Pós-Graduação em Gerontologia Biomédica. Pontifícia Universidade Católica do Rio Grande do Sul. Porto Alegre, RS, Brasil.

2 Instituto de Geriatria e Gerontologia. Pontifícia Universidade Católica do Rio Grande do Sul. Porto Alegre, RS, Brasil. 
was the one proposed by Beaton et al., with two translations to Portuguese followed by a synthesis of these two translations, two back translations, a synthesis of these back translations, an analysis by the specialist committee and a pretest. The validity of the content was performed by the analysis of the committee and the qualitative analysis of the pretest. After the application of the pretest and an individual interview with the applicants, two alterations were necessary on the document used as pretest. The final document originated will be applied in the near future to a larger sample of individuals for its validation to the Brazilian population.

\section{INTRODUÇÃO}

O processo do envelhecimento é inerente a todo ser humano; no entanto, pode ser influenciado por hábitos saudáveis e atitudes positivas. A manutenção da funcionalidade física, psíquica e social é imprescindível para que o envelhecimento seja o mais ativo, autônomo e independente possível. ${ }^{1}$

A importância da autopercepção em relação a situações mais objetivas tem estimulado pesquisas na área gerontológica. Estudos têm demonstrado que uma percepção ou atitude mais positiva em relação ao envelhecimento está associada a benefícios tanto na saúde física como no bemestar emocional dos indivíduos. ${ }^{2}$ Fatores sociais, como relacionamentos com familiares e amigos, promovem uma autopercepção positiva tanto de saúde como de envelhecimento. Indivíduos que cultivam uma rede de relacionamentos apresentam uma percepção mais favorável deles mesmos e de sua situação de saúde. ${ }^{3} \mathrm{O}$ apoio de grupos sociais estimula positivamente a autoestima, contribuindo para uma percepção mais positiva do envelhecimento e saúde.

Poucos estudos, no entanto, têm focado nos aspectos psicológicos e sociais que podem também influenciar na promoção de incapacidades e interferir negativamente no processo de envelhecimento ativo e bemsucedido. Entre os fatores sociopsicológicos que podem ser perfeitamente modificados, está a percepção sobre o envelhecimento, isto é, como o indivíduo sente o processo de seu próprio envelhecimento. ${ }^{1,4}$
A percepção que um indivíduo tem de seu próprio envelhecimento pode contribuir sobremaneira para o sucesso deste processo. Vários estudos ${ }^{5,6,13}$ demonstraram que percepções positivas e negativas podem influenciar na recuperação de algumas doenças e na prevenção de outras. Esta percepção sobre o envelhecer está presente em todas as idades em maior ou menor grau de consciência, contribuindo diferentemente para a adaptação ao envelhecer. Estudos como os de Levy e colaboradores 4,6,13,14 têm demonstrado que associações negativas e positivas com o envelhecimento podem causar um impacto tanto emocional quanto cognitivo em indivíduos idosos ou naqueles que estejam chegando à velhice.

A autopercepção do envelhecimento parece ser um bom indicador de um envelhecimento bem-sucedido. ${ }^{5}$ A existência de problemas de saúde não apenas provoca um efeito negativo direto na percepção sobre o envelhecimento, como também indireto, através de uma percepção negativa sobre a saúde. Portanto, indivíduos idosos com um estado de saúde comprometido podem apresentar uma percepção negativa em relação à sua saúde, o que pode gerar uma percepção negativa sobre o envelhecimento como um todo. Barker et al. ${ }^{7}$ afirmaram que quanto melhor a saúde de um indivíduo idoso, percebida por ele mesmo, melhor sua autopercepção de envelhecimento.

Portanto, a autopercepção do envelhecimento é um bom indicador de um envelhecimento bem-sucedido. ${ }^{5}$ Estudos abordando a percepção sobre envelhecimento mostram que uma idade 
subjetiva jovem e uma alta satisfação com o envelhecimento estão associadas à boa saúde, altos níveis de bem-estar e poucos sintomas clínicos. ${ }^{6}$ Entretanto, no Brasil ainda não existem instrumentos e escalas validados e adaptados culturalmente para se avaliar especificamente a autopercepção do envelhecimento. Por isso, a necessidade de desenvolver e divulgar pesquisas sobre validação e propriedades psicométricas de escalas traduzidas e adaptadas ao nosso idioma e ao nosso país é fundamental.

Desta forma, o presente artigo visa a apresentar a tradução e adaptação cultural para a língua portuguesa do Brasil do Aging Perceptions Questionnaire (APQ), originalmente desenvolvido, validado e publicado em língua inglesa.

O APQ foi desenvolvido por Barker et al. ${ }^{7}$ na Irlanda e é um instrumento multidimensional autoaplicável que tem como objetivo avaliar a autopercepção do envelhecimento a partir do Modelo de Autorregulação (SRM). A base deste modelo é que um indivíduo forma uma representação de sua doença ou ameaça à saúde que pode ser dividido em uma série de dimensões lógicas: identidade (crenças sobre a natureza de sua doença e a relação desta com seus sintomas), cronologia (crenças sobre a duração de sua doença, se esta é aguda, crônica ou cíclica), as consequências (crenças sobre o impacto que esta doença tem na sua vida), controle (crenças sobre a maneira de gerenciar essa doença), causas (crenças sobre as possíveis causas da doença) e as representações emocionais (as respostas emocionais geradas pela doença).

Esse questionário avalia, portanto, a autopercepção do envelhecimento a partir de oito domínios distintos, sete dos quais envolvem opiniões sobre o próprio envelhecimento, enquanto que o oitavo é chamado domínio da identidade e examina a experiência dos indivíduos com as alterações em relação às doenças. Para a pontuação, é utilizada a escala de Likert, ${ }^{15}$ com escores de 1 a 5. O APQ apresenta duas partes: a primeira, composta por 32 itens, avalia a opinião do indivíduo sobre seu envelhecimento; a segunda, composta por 17 itens, avalia a existência de doença e sua relação com o processo de envelhecimento.

O processo de tradução e adaptação cultural de um instrumento é de suma importância, especialmente na área da saúde. Este processo exige, além da equivalência linguística, sua adaptação semântica, cultural e conceitual para que possa refletir com maior veracidade e confiabilidade os resultados propostos pelo instrumento original na nova população em estudo. Nascimento e Figueiredo ${ }^{8}$ referem que a utilização de um instrumento estrangeiro sem a sua devida adaptação pode colocar em risco a validade e a precisão dos resultados obtidos nestas avaliações. No entanto, relataram também a escassez de referências práticas sobre os procedimentos e análises envolvidas na construção e adaptação de instrumentos psicológicos.

Dessa forma, a tradução de uma escala deve primar pela linguagem simples e clara, mantendo-se equivalente no que tange a seus conceitos culturais e sociais, além da equivalência semântica e idiomática. ${ }^{9}$ Neste contexto, um dos protocolos de tradução e adaptação cultural mais amplamente utilizados no Brasil é o proposto por Beaton et al..$^{10-12}$

\section{MÉTODO}

O método para tradução e adaptação cultural utilizado no presente estudo foi o proposto por Beaton et al. ${ }^{12}$ Inicialmente, foi solicitada a autorização formal dos autores do questionário original, bem como obtida a aprovação da Comissão Científica e do Comitê de Ética em Pesquisa do Instituto de Geriatria e Gerontologia da PUCRS (Ofício n. 1.122/09 do CEP-PUC-RS).

\section{Procedimento}

Os procedimentos adotados neste estudo seguem as recomendações de Beaton et al. ${ }^{12} \mathrm{~A}$ figura 1 sintetiza tais recomendações. 


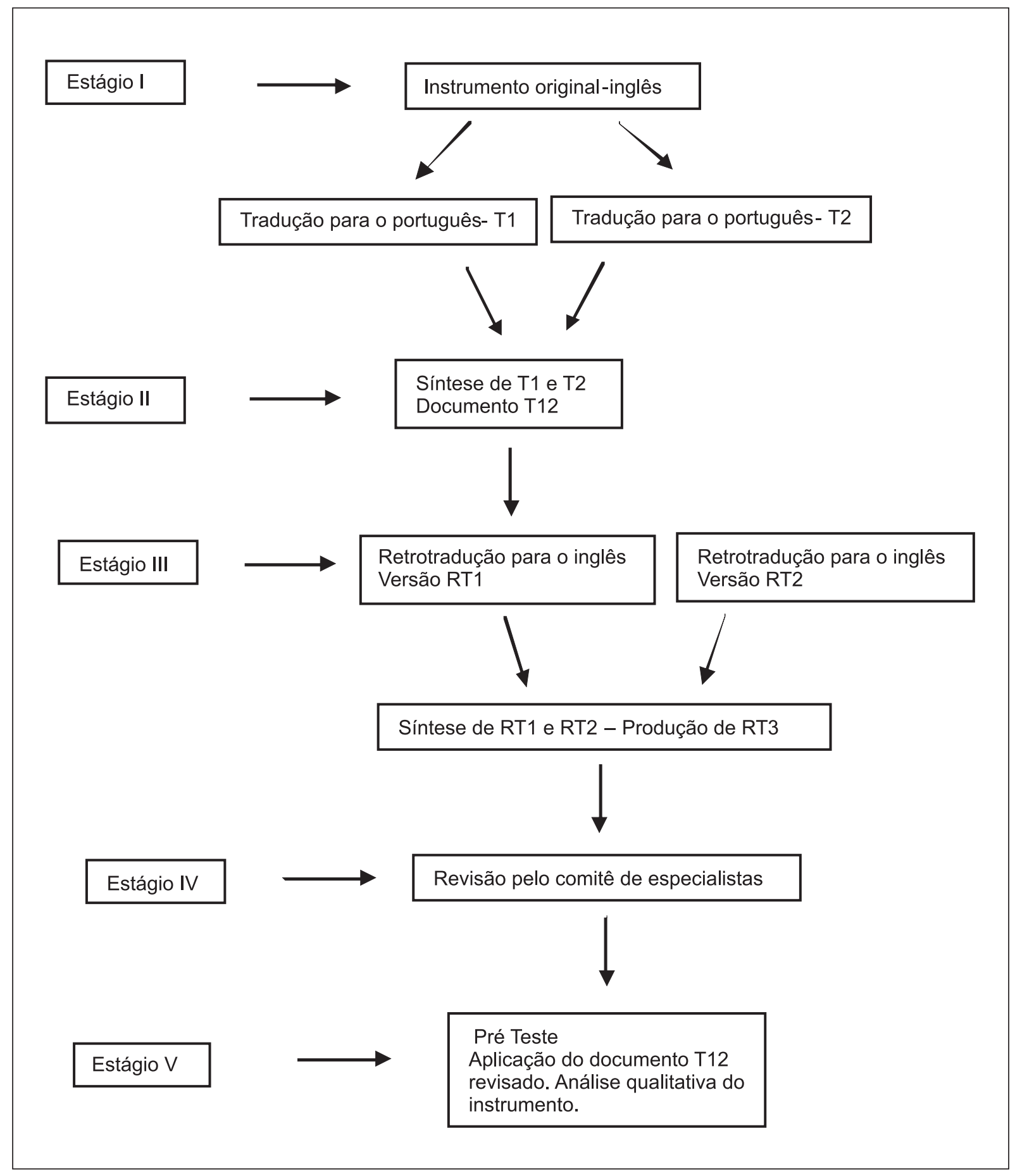

Figura 1 - Procedimentos para tradução e adaptação cultural conforme as recomendações do protocolo de Beaton et al. ${ }^{12}$ 
Primeiramente, o instrumento foi traduzido do inglês para o português por dois tradutores brasileiros com fluência na língua inglesa. Desta forma, foram gerados os documentos T1 e T2, respectivamente.

Após as traduções, os dois documentos (T1 e T2) foram submetidos ao comitê de especialistas composto por um epidemiologista, por um profissional médico e pelo tradutor médico, todos bilíngues que, conjuntamente, elaboraram a síntese da T1 e T2 - o documento T12 - que, submetida ao segundo tradutor, obteve sua aprovação.

O documento T12 foi então enviado a dois outros tradutores nativos da língua inglesa, ambos fluentes em português do Brasil, para as retrotraduções. Foram criados nesta etapa dois novos documentos, RT1 e RT2. Um dos tradutores reuniu-se com a pesquisadora $\mathrm{e}$ tradutora médica para elaborar a síntese das duas retrotraduções e elaborar o documento RT3. Palavras e expressões que diferiam entre as duas retrotraduções foram sublinhadas e analisadas posteriormente quanto a melhor escolha para o documento final. Após o acordo entre os dois tradutores, o documento RT3 foi remetido ao comitê de especialistas para análise e comparação ao documento original. Todos os membros do comitê de especialistas eram fluentes em inglês e português. Neste momento, o documento original, a versão T12 e a RT3 foram comparadas pelo comitê para análise semântica, idiomática, conceitual e cultural.

Após a etapa de retrotradução, foi realizado o pré-teste com 30 indivíduos com idade igual ou superior a 60 anos, pacientes do ambulatório de Geriatria do Hospital São Lucas da PUC-RS, escolhidos por conveniência, que responderam ao questionário T12, após as alterações do comitê de especialistas. Todos participantes assinaram um termo de consentimento livre e esclarecido. A seguir, cada participante foi entrevistado individualmente pela pesquisadora quanto ao entendimento do questionário e possíveis dúvidas que tiveram para responder às questões do instrumento. O objetivo principal do pré-teste foi fazer uma avaliação qualitativa do instrumento como parte do processo de adaptação cultural à nova população para a qual se destina.

\section{RESULTADOS}

Para o pré-teste foram entrevistados 30 indivíduos do ambulatório de Geriatria do Hospital São Lucas da PUC-RS com idade variando entre 62 e 100 anos (76,48 \pm 9,26), de ambos os sexos, tendo sido observada uma predominância do sexo feminino $(92,6 \%)$ sobre o sexo masculino (7,4\%), o que confirma a tendência da feminização do envelhecimento. Dentre os entrevistados, 11,1 eram analfabetos e $33,3 \%$ não completaram o ensino fundamental. Os resultados obtidos mostraram que o APQ, em sua versão para o português do Brasil, apresentou uma equivalência cultural e conceitual ao instrumento original, não sendo necessárias adaptações neste âmbito (quadro 1).

Quanto ao quesito semântico, foram realizadas alterações em dois itens do questionário a partir da análise das entrevistas com os pacientes. O item 15 "Whether getting older has positive sides to it depends on me", traduzido no documento T12 utilizado para o pré-teste por "Se o fato de envelhecer tem aspectos positivos é algo que depende de mim", foi alterado para "Depende de mim o fato de envelhecer ter aspectospositivos", visto ter gerado problemas de entendimento em mais de um entrevistado no pré-teste. No item 16, a palavra "restringe" foi substituída por "limita" para melhor entendimento da afirmação, após a aplicação do pré-teste e análise qualitativa do mesmo. Estas alterações deram origem à versão final do instrumento traduzido para a língua portuguesa brasileira.

Adicionalmente, a maior parte dos sujeitos de pesquisa $(70 \%)$ necessitou de auxílio do entrevistador para ler as perguntas, a maioria por problemas visuais. Não foi observada, no entanto, dificuldade em escolher as respostas. O tempo médio de aplicação do APQ foi de 15 minutos. 
Quadro 1 - Tradução e adaptação cultural do Aging Perceptions Questionnaire (APQ) para a língua portuguesa brasileira

\section{QUESTIONÁRIO SOBRE PERCEPCÕES DE ENVELHECIMENTO (APQ)}

As questões abaixo avaliam suas opiniões e experiências sobre o processo de envelhecimento. Como todos estão envelhecendo, estas questões podem ser respondidas por qualquer pessoa de qualquer idade. Não existem respostas certas ou erradas, apenas suas experiências e opiniões. Mesmo que a questão seja sobre um assunto que você não pense com frequência em relação a si mesmo, favor tentar dar uma ideia do que pensa, respondendo a todas as perguntas.

\begin{tabular}{|c|c|c|c|c|c|}
\hline \multicolumn{6}{|c|}{ A) OPINIÕES SOBRE O ENVELHECIMENTO } \\
\hline \multicolumn{6}{|c|}{$\begin{array}{l}\text { Estamos interessados nas suas opiniões e experiências pessoais sobre o processo de envelhecimento. Indique suas } \\
\text { opiniões sobre as afirmações seguintes (discordo totalmente, discordo, não concordo nem discordo, concordo ou } \\
\text { concordo plenamente). Circule a resposta que melhor descreve sua opinião sobre cada afirmação. }\end{array}$} \\
\hline & $\begin{array}{l}\text { Discordo } \\
\text { Totalmente }\end{array}$ & Discordo & $\begin{array}{l}\text { Não } \\
\text { concordo } \\
\text { nem } \\
\text { discordo }\end{array}$ & Concordo & $\begin{array}{l}\text { Concordo } \\
\text { Plenamente }\end{array}$ \\
\hline $\begin{array}{l}\text { 1. Tenho consciência de estar envelhecendo } \\
\text { o tempo todo }\end{array}$ & $\square 1$ & $\square 2$ & $\square 3$ & $\square 4$ & $\square 5$ \\
\hline 2. Estou sempre consciente da minha idade & $\square 1$ & $\square 2$ & $\square 3$ & $\square 4$ & $\square 5$ \\
\hline 3. Sempre me classifico como velho/a & $\square 1$ & $\square 2$ & $\square 3$ & $\square 4$ & $\square 5$ \\
\hline $\begin{array}{l}\text { 4. Estou sempre consciente do fato de que } \\
\text { estou envelhecendo }\end{array}$ & $\square 1$ & $\square 2$ & $\square 3$ & $\square 4$ & $\square 5$ \\
\hline 5. Sinto minha idade em tudo que faço & $\square 1$ & $\square 2$ & $\square 3$ & $\square 4$ & $\square 5$ \\
\hline $\begin{array}{l}\text { 6. À medida que envelheço, vou ganhando } \\
\text { sabedoria }\end{array}$ & $\square 1$ & $\square 2$ & $\square 3$ & $\square 4$ & $\square 5$ \\
\hline $\begin{array}{l}\text { 7. À medida que envelheço, continuo } \\
\text { crescendo como pessoa }\end{array}$ & $\square 1$ & $\square 2$ & $\square 3$ & $\square 4$ & $\square 5$ \\
\hline $\begin{array}{l}\text { 8. À medida que envelheço, aprecio mais as } \\
\text { coisas }\end{array}$ & $\square 1$ & $\square 2$ & $\square 3$ & $\square 4$ & $\square 5$ \\
\hline $\begin{array}{l}\text { 9. Fico deprimido/a quando penso sobre } \\
\text { como o envelhecimento pode afetar as } \\
\text { coisas que eu consigo fazer }\end{array}$ & $\square 1$ & $\square 2$ & $\square 3$ & $\square 4$ & $\square 5$ \\
\hline $\begin{array}{l}\text { 10. A qualidade da minha vida social na } \\
\text { velhice depende de mim }\end{array}$ & $\square 1$ & $\square 2$ & $\square 3$ & $\square 4$ & $\square 5$ \\
\hline $\begin{array}{l}\text { 11. A qualidade de meus relacionamentos } \\
\text { com os outros na velhice depende de mim }\end{array}$ & $\square 1$ & $\square 2$ & $\square 3$ & $\square 4$ & $\square 5$ \\
\hline $\begin{array}{l}\text { 12. Se eu vou continuar vivendo a vida } \\
\text { plenamente é algo que depende de mim }\end{array}$ & $\square 1$ & $\square 2$ & $\square 3$ & $\square 4$ & $\square 5$ \\
\hline $\begin{array}{l}\text { 13. Fico deprimido/a quando penso como o } \\
\text { envelhecimento pode afetar minha vida } \\
\text { social }\end{array}$ & $\square 1$ & $\square 2$ & $\square 3$ & $\square 4$ & $\square 5$ \\
\hline $\begin{array}{l}\text { 14. À medida que vou envelhecendo, há } \\
\text { muito que posso fazer para manter } \\
\text { minha independência }\end{array}$ & $\square 1$ & $\square 2$ & $\square 3$ & $\square 4$ & $\square 5$ \\
\hline $\begin{array}{l}\text { 15. Depende de mim o fato de envelhecer } \\
\text { ter aspectos positivos. }\end{array}$ & $\square 1$ & $\square 2$ & $\square 3$ & $\square 4$ & $\square 5$ \\
\hline
\end{tabular}


Quadro 1 - Tradução e adaptação cultural do Aging Perceptions Questionnaire (APQ) para a língua portuguesa brasileira (Continuação)

\begin{tabular}{|c|c|c|c|c|c|}
\hline \multicolumn{6}{|c|}{ A) OPINIÕES SOBRE O ENVELHECIMENTO } \\
\hline \multicolumn{6}{|c|}{$\begin{array}{l}\text { Estamos interessados nas suas opiniões e experiências pessoais sobre o processo de envelhecimento. Indique suas } \\
\text { opiniões sobre as afirmações seguintes (discordo totalmente, discordo, não concordo nem discordo, concordo ou } \\
\text { concordo plenamente). Circule a resposta que melhor descreve sua opinião sobre cada afirmação. }\end{array}$} \\
\hline & $\begin{array}{l}\text { Discordo } \\
\text { Totalmente }\end{array}$ & Discordo & $\begin{array}{c}\text { Não } \\
\text { concordo } \\
\text { nem } \\
\text { discordo }\end{array}$ & Concordo & $\begin{array}{l}\text { Concordo } \\
\text { Plenamente }\end{array}$ \\
\hline $\begin{array}{l}\text { 16. Envelhecer limita as coisas que posso } \\
\text { fazer }\end{array}$ & $\square 1$ & $\square 2$ & $\square 3$ & $\square 4$ & $\square 5$ \\
\hline 17. Envelhecer me torna menos independente & $\square 1$ & $\square 2$ & $\square 3$ & $\square 4$ & $\square 5$ \\
\hline $\begin{array}{l}\text { 18. Envelhecer torna tudo muito mais difícil } \\
\text { para mim }\end{array}$ & $\square 1$ & $\square 2$ & $\square 3$ & $\square 4$ & $\square 5$ \\
\hline $\begin{array}{l}\text { 19. À medida que envelheço, posso } \\
\text { participar de cada vez menos atividades }\end{array}$ & $\square 1$ & $\square 2$ & $\square 3$ & $\square 4$ & $\square 5$ \\
\hline $\begin{array}{l}\text { 20. À medida que envelheço, já não lido tão } \\
\text { bem com os problemas que surgem }\end{array}$ & $\square 1$ & $\square 2$ & $\square 3$ & $\square 4$ & $\square 5$ \\
\hline $\begin{array}{l}\text { 21. A diminuição do ritmo de vida com a } \\
\text { idade não é algo que eu possa controlar }\end{array}$ & $\square 1$ & $\square 2$ & $\square 3$ & $\square 4$ & $\square 5$ \\
\hline $\begin{array}{l}\text { 22. A minha mobilidade ao envelhecer não } \\
\text { depende de mim }\end{array}$ & $\square 1$ & $\square 2$ & $\square 3$ & $\square 4$ & $\square 5$ \\
\hline $\begin{array}{l}\text { 23. Não tenho controle sobre a perda de } \\
\text { vitalidade ou de entusiasmo pela vida à } \\
\text { medida que vou envelhecendo }\end{array}$ & $\square 1$ & $\square 2$ & $\square 3$ & $\square 4$ & $\square 5$ \\
\hline $\begin{array}{l}\text { 24. Não tenho controle sobre os efeitos que } \\
\text { o envelhecimento exerce sobre minha } \\
\text { vida social }\end{array}$ & $\square 1$ & $\square 2$ & $\square 3$ & $\square 4$ & $\square 5$ \\
\hline $\begin{array}{l}\text { 25. Fico deprimido/a quando penso em } \\
\text { envelhecer }\end{array}$ & $\square 1$ & $\square 2$ & $\square 3$ & $\square 4$ & $\square 5$ \\
\hline $\begin{array}{l}\text { 26. Eu me preocupo com os efeitos que o } \\
\text { envelhecimento pode ter sobre meus } \\
\text { relacionamentos com os outros }\end{array}$ & $\square 1$ & $\square 2$ & $\square 3$ & $\square 4$ & $\square 5$ \\
\hline $\begin{array}{l}\text { 27. Minha experiência com o envelhecimento } \\
\text { é cíclica às vezes piora e às vezes melhora. }\end{array}$ & $\square 1$ & $\square 2$ & $\square 3$ & $\square 4$ & $\square 5$ \\
\hline $\begin{array}{l}\text { 28. Minha consciência de estar } \\
\text { envelhecendo vai e volta em ciclos }\end{array}$ & $\square 1$ & $\square 2$ & $\square 3$ & $\square 4$ & $\square 5$ \\
\hline $\begin{array}{l}\text { 29. Fico brabo/a quando penso em } \\
\text { envelhecer }\end{array}$ & $\square 1$ & $\square 2$ & $\square 3$ & $\square 4$ & $\square 5$ \\
\hline 30. Passo por fases em que me sinto velho/a & $\square 1$ & $\square 2$ & $\square 3$ & $\square 4$ & $\square 5$ \\
\hline $\begin{array}{l}\text { 31. Minha consciência de estar envelhecendo } \\
\text { muda muito de um dia para outro }\end{array}$ & $\square 1$ & $\square 2$ & $\square 3$ & $\square 4$ & $\square 5$ \\
\hline $\begin{array}{l}\text { 32. Passo por fases em que me vejo como } \\
\text { velho/a }\end{array}$ & $\square 1$ & $\square 2$ & $\square 3$ & $\square 4$ & $\square 5$ \\
\hline
\end{tabular}


Quadro 1 - Tradução e adaptação cultural do Aging Perceptions Questionnaire (APQ) para a língua portuguesa brasileira (Continuação)

\section{B) EXPERIÊNCIA COM MUDANÇAS RELACIONADAS À SAÚDE}

A lista a seguir descreve algumas mudanças relacionadas à saúde que talvez você tenha vivenciado. Pode me dizer se vivenciou essas mudanças nos últimos 10 anos e se acredita que as mudanças vivenciadas estão especificamente relacionadas com o fato de envelhecer?

\begin{tabular}{|l|l|c|c|c|c|}
\hline & \multicolumn{3}{|c|}{$\begin{array}{c}\text { Você VIVENCIOU } \\
\text { esta mudança? }\end{array}$} & $\begin{array}{r}\text { 'Em termos das mudanças que você } \\
\text { VIVENCIOU: Acha que esta mudança está } \\
\text { relacionada ou deve-se SOMENTE ao fato } \\
\text { de você estar envelhecendo? }\end{array}$ \\
\hline & & Sim & Não & Sim & Não \\
\hline Id1 & Problemas com o peso & $\square 1$ & $\square 0$ & $\square 1$ & $\square 0$ \\
\hline Id2 & Problemas com o sono & $\square 1$ & $\square 0$ & $\square 1$ & $\square 0$ \\
\hline Id3 & $\begin{array}{l}\text { Problemas nas costas ou hérnia de } \\
\text { disco }\end{array}$ & $\square 1$ & $\square 0$ & $\square 1$ & $\square 0$ \\
\hline Id4 & Dor nas articulações & $\square 1$ & $\square 0$ & $\square 1$ & $\square 0$ \\
\hline Id5 & Não ter mobilidade & $\square 1$ & $\square 0$ & $\square 1$ & $\square 0$ \\
\hline Id6 & Perda de equilíbrio & $\square 1$ & $\square 0$ & $\square 1$ & $\square 0$ \\
\hline Id7 & Perda de força & $\square 1$ & $\square 0$ & $\square 1$ & $\square 0$ \\
\hline Id8 & Redução do ritmo de vida & $\square 1$ & $\square 0$ & $\square 1$ & $\square 0$ \\
\hline Id9 & Câimbras & $\square 1$ & $\square 0$ & $\square 1$ & $\square 0$ \\
\hline Id10 & Problemas nos ossos ou articulações & $\square 1$ & $\square 0$ & $\square 1$ & $\square 0$ \\
\hline Id11 & Problemas cardíacos & $\square 1$ & $\square 0$ & $\square 1$ & $\square 0$ \\
\hline Id12 & Problemas de audição & $\square 1$ & $\square 0$ & $\square 1$ & $\square 0$ \\
\hline Id13 & Mudanças na visão & $\square 1$ & $\square 0$ & $\square 1$ & $\square 0$ \\
\hline Id14 & Problemas respiratórios & $\square 1$ & $\square 0$ & $\square 1$ & $\square 0$ \\
\hline Id15 & Problemas nos pés & $\square 1$ & $\square 0$ & $\square 1$ & $\square 1$ \\
\hline Id16 & Depressão & $\square 0$ & $\square 0$ & $\square 1$ & $\square 1$ \\
\hline Id17 & Ansiedade & $\square 1$ & $\square 0$ \\
\hline
\end{tabular}




\section{CONSIDERAÇÕES FINAIS}

O processo de tradução e adaptação de um instrumento é extremamente complexo, assemelhando-se a criação e desenvolvimento de um novo instrumento. Neste processo, deve-se ter em mente a busca de equivalências linguísticas que proporcionem a utilização do instrumento na nova cultural sem perda de sua eficácia. ${ }^{15}$

A importância de estudos transculturais na área da saúde e, em especial, sobre o processo do envelhecimento, reforça a necessidade de tradução e adaptação de instrumentos muitas vezes de origem na língua inglesa para outras línguas e culturas.

\section{REFERÊNCIAS}

1. Kempen GI, van Heuvelen MJ, van Sonderen E, van den Brink RH, Kooijman AC, Ormel J. The relationship of functional limitations to disability and the moderating effects of psychological attributes in community-dwelling older persons. Soc Sci Med. 1999 May; 48(9):1161-72.

2. Maier H, Smith J. Psychological predictors of mortality in old age. J Gerontol B Psychol Sci Soc Sci. 1999 Jan;54(1):P44-54.

3. Krause N. Social support and feelings of personal control in later life. In: Pierce G, Lakey B, Sarason I, Sarason B (Eds.). Sourcebook of social support and personality. New York: Plenum Press; 1997. p. 335-55.

4. Levy B, Myers LM. Preventive health behaviors influenced by self-perceptions of aging. Prev Med. 2004 Sep;39(3):625-9.

5. Baltes PB, Smith J. New frontiers in the future of aging: From successful aging of the young old to the dilemmas of the fourth age. Gerontology 2003; 49:123-35.
Os resultados destas pesquisas podem auxiliar no entendimento das várias imagens e percepções do processo do envelhecimento e estimular novas pesquisas nesta área, visto que estamos beirando uma nova identidade populacional na maioria dos países do continente sul-americano. Observa-se que a população está cada vez mais velha e demanda mais atenção, principalmente no que se refere à prevenção de agravos.

Concluindo, a versão para o português do APQ foi obtida por meio de um cuidadoso processo de tradução e adaptação, compatível com as recomendações previstas atuais para esse processo $^{12}$. A próxima etapa do estudo envolverá a validação do instrumento traduzido e adaptado para a população brasileira.

6. Levy BR, Slade M, Kasl SV. Longitudinal benefit of positive self-perceptions of aging on functional health. J Gerontol B Psychol Sci Soc Sci. 2002 Sep;57(5):P409-17.

7. Barker M, O'Hanlon A, McGee HM, Hickey A, Conroy RM. Cross-sectional validation of the Aging Perceptions Questionnaire: a multidimensional instrument for assessing self-perceptions of aging. BMC Geriatr 2007;7:9.

8. Nascimento E, Figueiredo VL. WISC-III e WAISIII: alterações nas versões originais americanas decorrentes das adaptações para uso no Brasil. Psicologia: Reflexão e Crítica. 2002;15(3):603-12

9. Ciconelli R.M. Tradução para a língua portuguesa e validação do questionário genérico de avaliação de qualidade de vida SF-36(Brasil SF36). Rev Bras Reumatol. 1999; 39( 3):143-50.

10. Santos ALP, Simões AC. Tradução e adaptação transcultural do Physical Activity Scale de Aadahal e Jorgensen. Revista brasileira de educação física e esporte 2009 abr./jun.; 23(2):143-53. 
11. Giusti E, Befi-Lopes DM. Tradução e adaptação transcultural de instrumentos estrangeiros para o Português Brasileiro (PB). Pro Fono. 2008 julset;20(3):207-10.

12. Beaton D. Bombardier C. Guillemin F.,Ferraz M. Recommendations for the Cross-Cultural Adaptation of Health Status Measures of the American Academy of Orthopaedic Surgeons (AAOS). 2002. Disponível em: http:/www.dash.iwh.on.ca/assets/images/pdfs/XCulturalAdaptation-2007.pdf. Acesso em 10/04/2010.
13. Levy BR, et al. Longevity increased by positive self-perceptions of aging. J Pers Soc Psychol. 2002; 83(2): 261-70.

14. Levy BR, Langer EJ. Aging free from negative stereotypes: Successful memory in China and among the American deaf. J Pers Soc Psychol.1994; 66(6):989-97.

15. Freitas S, et al. Tradução da "Binge Eating Scale". Rev Bras Psiquiatr. 2001; 23 (4) :215-20.

16. Likert R. A Technique for the Measurement of Attitudes. Archives of psychology. 1932; 22(140); 1-55.

Recebido: $17 / 11 / 2010$

Revisado: $02 / 3 / 2011$

Aprovado: 22/8/2011 